\title{
A Modified Iterative Alternating Direction Minimization Algorithm for Impulse Noise Removal in Images
}

\author{
Di Guo, ${ }^{1}$ Xiaobo Qu, ${ }^{2}$ Meng $W u,{ }^{3}$ and Keshou $W u^{1}$ \\ ${ }^{1}$ School of Computer and Information Engineering, Xiamen University of Technology, Xiamen 361024, China \\ ${ }^{2}$ Department of Electronic Science, Xiamen University, Xiamen 361005, China \\ ${ }^{3}$ School of Information and Control Engineering, Xi'an University of Architecture and Technology, Xi'an 710055, China
}

Correspondence should be addressed to Di Guo; guodi@xmut.edu.cn

Received 10 May 2014; Revised 16 June 2014; Accepted 16 June 2014; Published 6 July 2014

Academic Editor: Filomena Cianciaruso

Copyright (c) 2014 Di Guo et al. This is an open access article distributed under the Creative Commons Attribution License, which permits unrestricted use, distribution, and reproduction in any medium, provided the original work is properly cited.

\begin{abstract}
Images are often corrupted by impulse noise. In this paper, an alternating direction minimization with continuation algorithm is modified and iteratively used to remove the impulse noise in images by exploring its self-similarity. A patch-based nonlocal operator and sparse representation are married in the $l_{1}-l_{1}$ optimization model to be solved. Simulation results demonstrate that the proposed algorithm outperforms typical denoising methods in terms of preserving edges and textures for both salt-and-pepper noise and random-valued impulse noise. It can be also applied to suppress impulse noise-like artifacts in real mural images.
\end{abstract}

\section{Introduction}

Image denoising is a fundamental problem in image processing. Besides the commonly processed Gaussian noise, impulse noise (Figure 1(a)) is another typical type of noise generated due to noisy sensors or channel transmission errors [1]. In mural images, the impulse noise-like artifacts are also observed because of the bacterial plaque (Figure 1(b)) or missing objects (Figure 1(c)). A classical impulse denoising method is median filtering, which replaces the central pixel with a median-value within a local window. Median filtering is with low computation but often loses image structures when noise is heavy. The denoising performance is expected to be greatly improved if the image structures are extracted and preserved in denoising.

In recent years, sparse representation method has attracted great attention to provide effective representation of image structures and found promising applications in image denoising [2-5], image decomposition [6, 7], image fusion $[8,9]$, pattern recognition [10-13], and so forth. In impulse noise removal, sparse representation is remarkably effective at smoothing away noise [14-16], but it may lose image structures which are not sparsely represented by its predefined basis such as wavelet $[3,14]$. Although the adaptive dictionary training $[15,16]$ improves the denoising performance, its computation cost of training dictionaries while removing noise is relatively high. Besides, the commonly used total variation [17-20] is highly related to enforcing the sparsity of finite difference of images. However, total variation assumes that images are piecewise smooth; thus, textures would be easily lost after denoising $[15,16,21,22]$. Therefore, exploring the sparse representation for impulse noise removal is still challenging.

Similarity is often rooted in repeated patterns of images [23-25] and greatly improves image quality in deblurring [26] and medical image reconstruction [27]. Similarity information was previously investigated in block matching 3D frames [26]. Patch-based nonlocal operator (PANO) [27] is a linear operator to model the sparse representation of similar patches, which can be viewed as an alternative form of the block matching 3D frames. Unlike the traditional sparsifying methods, for example, conventional wavelets or finite difference, PANO provides an adaptive sparse representation of image with a very fast training phase. Besides, the linearity of PANO allows setting up explicitly a sparsity-based denoising model. 


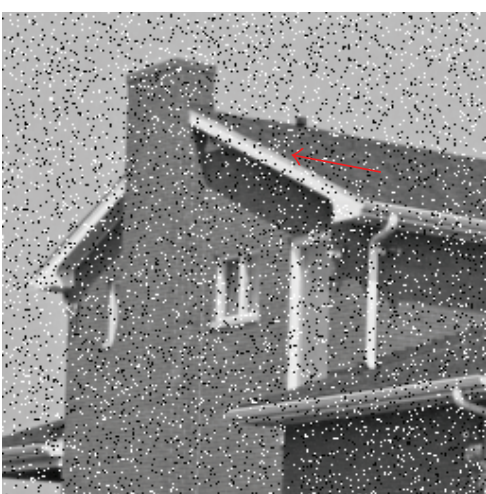

(a)

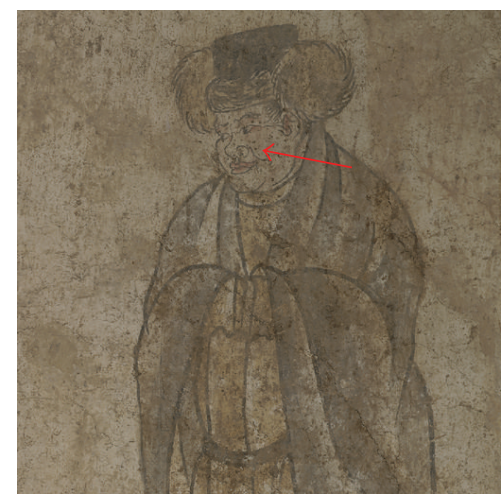

(b)

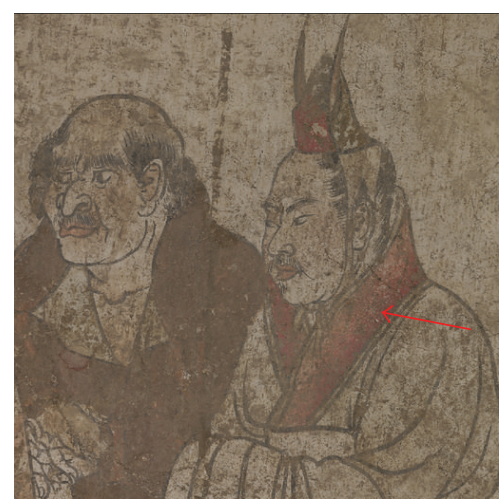

(c)

FIGURE 1: Images with impulse noise. (a) House image with 10\% salt-and-pepper noise; (b) and (c) are two mural images.

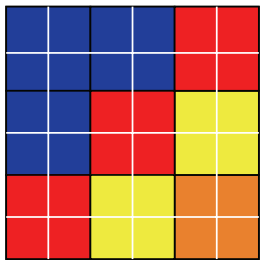

(a)

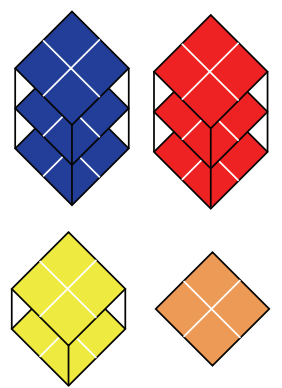

(b)

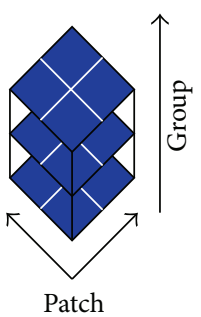

(c)

FIGURE 2: Group image patches. (a) An image; (b) four groups of similar patches; (c) the patch and group dimension.

TABLE 1: Effect of initial guide image with $50 \%$ salt-and-pepper noise in Barbara.

\begin{tabular}{lcccc}
\hline $\begin{array}{l}\text { Initial guide } \\
\text { image }\end{array}$ & $\begin{array}{c}\text { Median } \\
\text { filter }\end{array}$ & $\begin{array}{c}\text { Total } \\
\text { variation }\end{array}$ & Wavelet & Original \\
\hline PSNR & 32.42 & 32.40 & 32.45 & $\mathbf{3 3 . 0 9}$ \\
MSSIM & 0.7288 & 0.7280 & 0.7376 & $\mathbf{0 . 8 2 9 8}$ \\
\hline
\end{tabular}
lows.

The contributions of this paper are summarized as fol-

(1) The impulse noise is removed by making use of similarity-motivated sparse representation of images. Mathematically, we model the denoising problem as an $l_{1}-l_{1}$ minimization problem with PANO which provides the sparse representation of similar image patches.

(2) To solve the optimization problem, a fast alternating direction minimization with continuation algorithm is developed by taking the patch-based representation property into account.

(3) Besides removing salt-and-pepper noise and random-value impulse noise in images, the proposed method is also applied in recovering mural images from corrupted data. Experimental results on simulated data and real mural images verify that the proposed method outperforms other methods in removing impulse noise or impulse noise-like artifacts.

\section{Model}

In this section, the impulse noise model and typical denoising methods are briefly summarized. Then, the proposed denoising model is presented.

2.1. Impulse Noise. Image contaminated with impulse noise is usually modeled as

$$
\mathbf{f}=\boldsymbol{\eta} \odot \mathbf{u}+(\mathbf{1}-\boldsymbol{\eta}) \odot \varepsilon,
$$

where $\mathbf{u}$ is the noiseless image, $\mathbf{f}$ is the observation, $\odot$ denotes an element-wise product, $\boldsymbol{\eta} \in \mathbb{R}^{N}$ is a sample drawn from an i.i.d. multivariate Bernoulli distribution with success probability $1-p, \mathbf{1} \in \mathbb{R}^{N}$ represents a vector with all elements being equal to one, $\boldsymbol{\varepsilon} \in \mathbb{R}^{N}$ is either salt-and-pepper noise or random-valued impulse noise.

For the salt-and-pepper noise, the element $\varepsilon_{n}$ of $\varepsilon$ satisfies

$$
\varepsilon_{n}= \begin{cases}c_{\min } & \text { with probability } p_{1} \\ c_{\max } & \text { with probability } p_{2}\end{cases}
$$






(a)

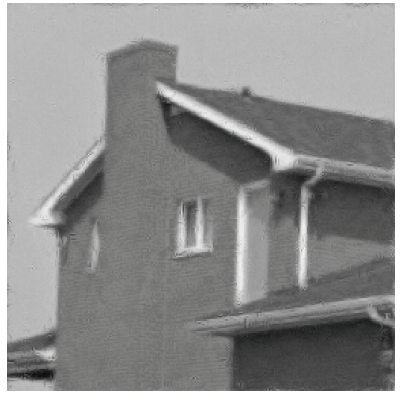

(e)

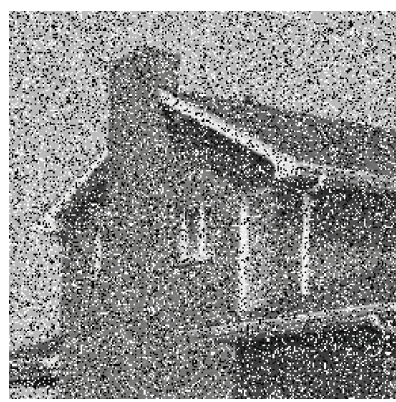

(b)

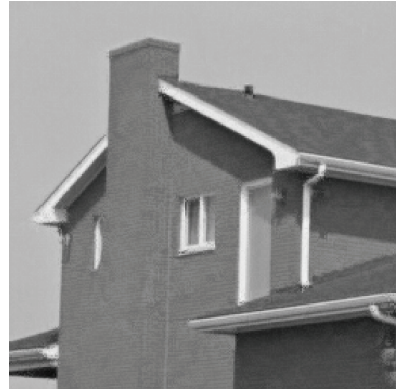

(f)



(i)

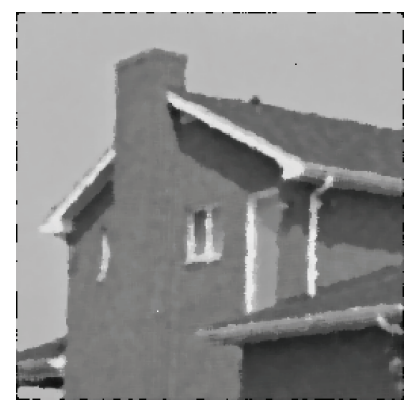

(c)



(g)

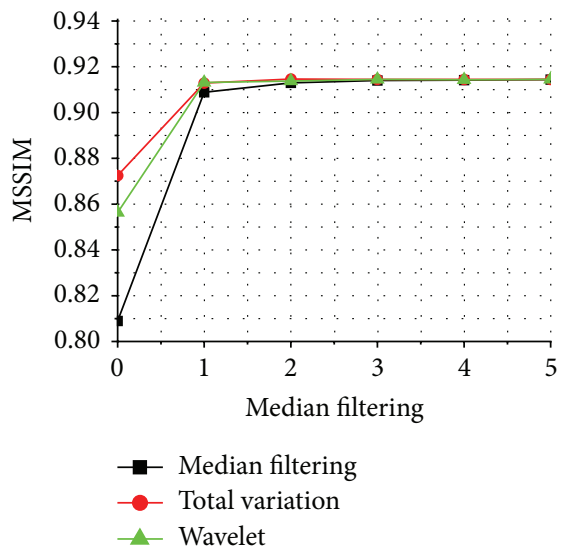

(j)



(d)

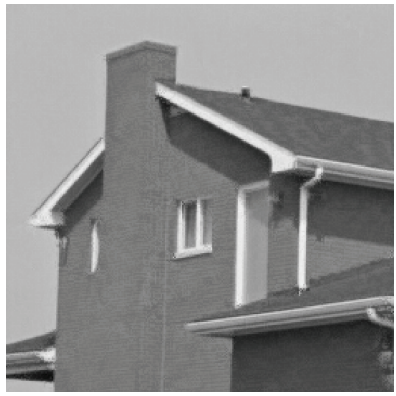

(h)

FIGURE 3: Effect of initial guide images on the denoising performance of the proposed method. (a) Original House image; (b) noisy image with 30\% salt-and-pepper noise; (c), (d), and (e) are denoised images using median filtering, total variation, and wavelet, respectively; (f), $(\mathrm{g})$, and (h) are denoised images using the proposed method with (c), (d), and (e) as the initial guide image, respectively; (i) and (j) are the PSNR and MSSIM performance curves.

where $p_{1}+p_{2}=p$. For the random-valued impulse noise, $\varepsilon_{n}$ is drawn from uniform distribution in $\left[c_{\min }, c_{\max }\right]$. In this paper, $c_{\min }=0, c_{\max }=1$, and $p_{1}=p_{2}$ are set.

\subsection{Proposed Denoising Model. With a maximum a posteriori} estimator on the impulse noise and prior knowledge of image, the noiseless image $\mathbf{u}$ is estimated by minimizing

$$
\min _{\mathbf{u}} \lambda\|\mathbf{f}-\mathbf{u}\|_{1}+\varphi(\mathbf{u}),
$$

where $\varphi$ can be chosen as total variation assuming that the image is piecewise constant $[19,28]$ or $\ell_{1}$ norm of wavelet coefficients assuming that the image is sparse in the wavelet domain [14]. However, total variation is observed to easily lose textures [16] and wavelet may lose image structures that are not sparsely represented by its predefined basis. Thus, how to preserve the edges and textures remains open for the impulse denoising problem.

In this paper, image self-similarity is employed to provide an optimized sparse representation of images. Assuming that the similarity information of image is known with PANO, an $\ell_{1}-\ell_{1}$ regularization model is proposed to remove the impulse noise,

$$
\min _{\mathbf{u}} \sum_{j=1}^{J}\left\|\mathbf{A}_{j} \mathbf{u}\right\|_{1}+\lambda\|\mathbf{f}-\mathbf{u}\|_{1}
$$






(a)

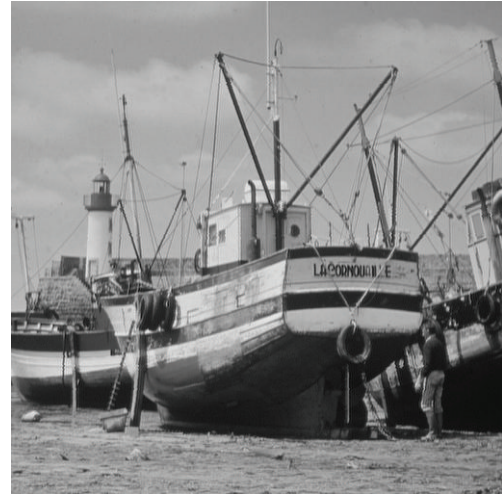

(d)

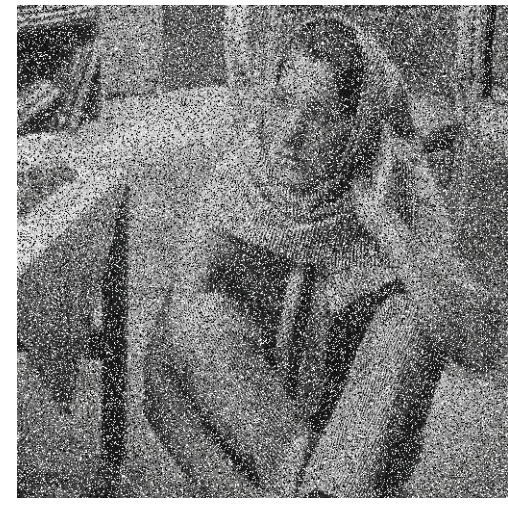

(b)

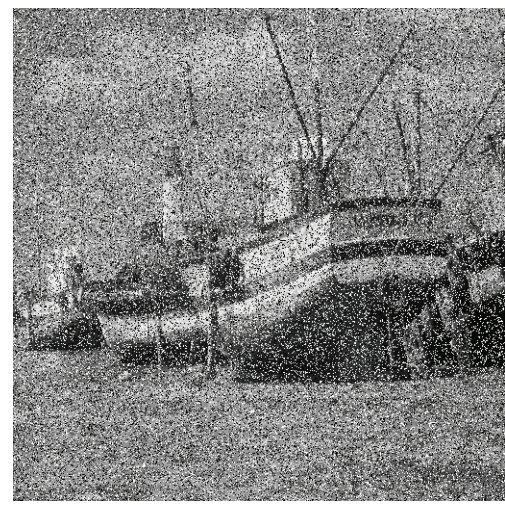

(e)

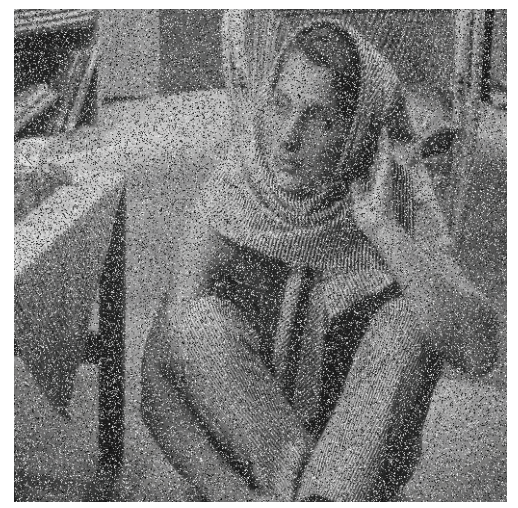

(c)



(f)

FIGURE 4: Images used in simulation. (a) and (d) are original images, Barbara (size $512 \times 512$ ) and Boat (size $512 \times 512$ ), respectively; (b) and (e) are the images corrupted with $30 \%$ salt-and-pepper noise; (c) and (f) are the images corrupted with $30 \%$ random-valued impulse noise.

\section{Initialization:}

Input the noise contaminated image $\mathbf{f}$, the PANO $\mathbf{A}_{j}(j=1, \ldots, J)$,

regularization parameter $\lambda$, tolerance of inner loop $\mu=10^{-3}$, and times of

learning similarity is 3. Initialize $\mathbf{u}=\mathbf{f}, \mathbf{z}=\mathbf{0}, \boldsymbol{\alpha}_{j}=\mathbf{0}$ for $j=1,2, \ldots, J, \beta=2^{4}$ and $\mathbf{u}_{\text {last }}=\mathbf{0}$.

Main:

Step 1. Obtain the initial guide image $\mathbf{r}$ from median filtering [1];

While $t \leq 3$,

Step 2. Learn similarity $\mathbf{A}_{j}(j=1, \ldots, J)$ in PANO from the guide image $\mathbf{r}$ [27];

While $\beta \leq 2^{14}$,

Step 3. Solve (8);

Step 4. Solve (9);

Step 5. Solve (10);

Step 6. If $\left\|\mathbf{u}_{\text {last }}-\mathbf{u}\right\|>\mu\left\|\mathbf{u}_{\text {last }}\right\|$, $\mathbf{u}_{\text {last }} \leftarrow \mathbf{u}$, go to Step 3; otherwise, go to Step 7;

End While

Step 7. $\beta \leftarrow 2 \beta, \widehat{\mathbf{u}} \leftarrow \mathbf{u}$, go to Step 3 .

End While

Step 8. $\mathbf{r} \leftarrow \widehat{\mathbf{u}}, t \leftarrow t+1, \beta=2^{4}$, Go to Step 2 ;

Output: $\widehat{\mathbf{u}}$

Algorithm 1: Remove impulse noise using PANO. 


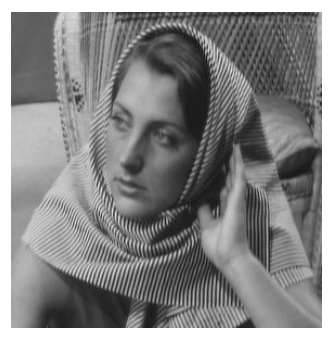

(a1)

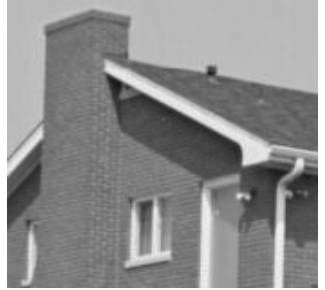

(a2)

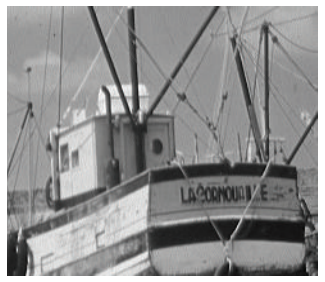

(a3)

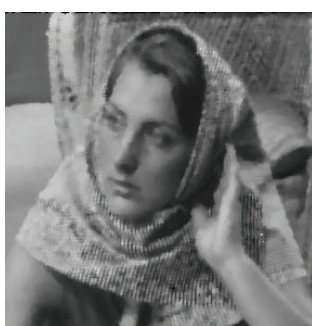

(b1)

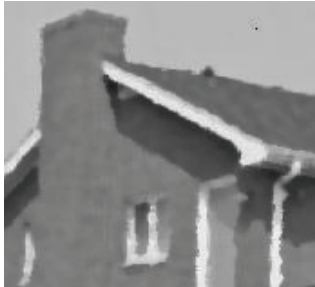

(b2)



(b3)

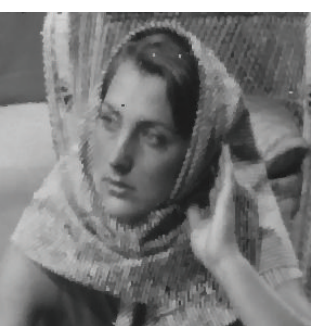

(c1)



(c2)

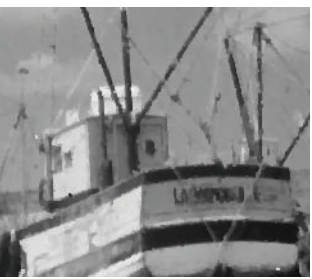

(c3)

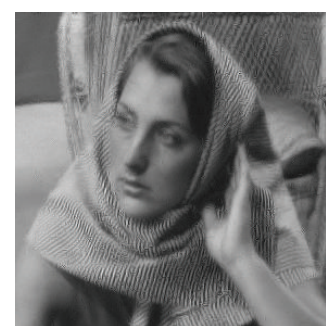

(d1)

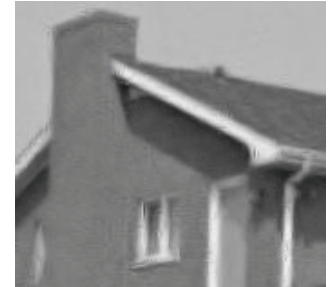

(d2)



(d3)

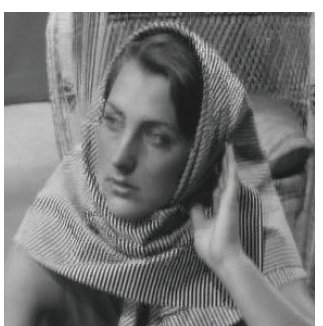

(e1)

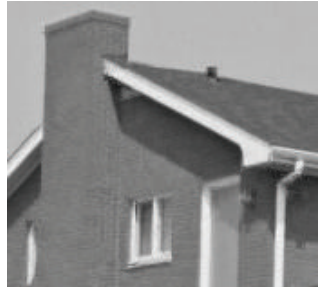

(e2)

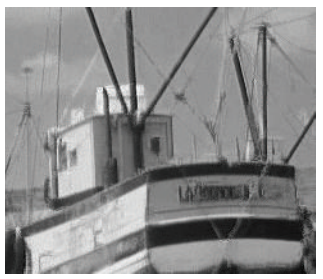

(e3)

Figure 5: Denoised images with 30\% salt-and-pepper noise. (a1), (a2), and (a3) are original images; (b1), (b2), and (b3) are results of median filtering; (c1), (c2), and (c3) are results of total variation denoising; (d1), (d2), and (d3) are results of the wavelet-based sparse representation denoising; (e1), (e2), and (e3) are results of the proposed method.

TABLE 2: Denoising performance of Barbara at high salt-and-pepper noise levels.

\begin{tabular}{|c|c|c|c|c|c|c|}
\hline \multirow{2}{*}{ Noise levels } & \multicolumn{2}{|c|}{ Proposed } & \multicolumn{2}{|c|}{ Total variation with detectors } & \multicolumn{2}{|c|}{ Proposed with detectors } \\
\hline & PSNR & MSSIM & PSNR & MSSIM & PSNR & MSSIM \\
\hline $50 \%$ & 32.47 & 0.7486 & 35.35 & 0.8647 & 36.18 & 0.9262 \\
\hline $70 \%$ & 30.36 & 0.6205 & 33.61 & 0.7790 & 33.49 & 0.8214 \\
\hline $90 \%$ & 26.09 & 0.4619 & 31.65 & 0.6310 & 30.54 & 0.6322 \\
\hline
\end{tabular}

where $\sum_{j=1}^{J}\left\|\mathbf{A}_{j} \mathbf{u}\right\|_{1}$ promotes the sparsity using PANO, $\|\mathbf{f}-\mathbf{u}\|_{1}$ removes the outliers in images, and $\lambda$ balances between the sparsity and outliers removing.

PANO is a linear operator to model the sparse representation of similar patches $[27,29]$. For a given image $\mathbf{u}$, PANO is defined as

$$
\mathbf{A}_{j}=\Psi_{3 \mathrm{D}} \mathbf{R}_{v_{j}} \mathbf{P}_{i}
$$

where $\mathbf{P}_{i}$ denotes the patch extraction, $\mathbf{R}_{v_{j}}$ means grouping some of the patches into a $3 \mathrm{D}$ cube, and $\Psi_{3 \mathrm{D}}$ is $3 \mathrm{D}$ Haar transform. Figure 2 illustrates the process of grouping patches into a cube. First, an image is extracted into patches. Then, some similar patches are grouped together and stacked into
3D cubes. Finally, a sparse representation will be achieved by performing a 3D Haar transform on each cube,

$$
\boldsymbol{\alpha}_{j}=\mathbf{A}_{j} \mathbf{u},
$$

where $\boldsymbol{\alpha}_{j}$ is the sparse vector for the $j$ th cube $[27,29]$. The adjoint operator of $\mathbf{A}_{j}$ is $\mathbf{A}_{j}^{T}=\mathbf{P}_{i}^{T} \mathbf{R}_{v_{j}}^{T} \boldsymbol{\Psi}_{3 \mathrm{D}}^{T}$, and $\sum_{j}^{J} \mathbf{A}_{j}^{T} \mathbf{A}_{j}=$ $\mathbf{O}$, where $\mathbf{O}$ is a diagonal matrix whose $n$th diagonal entry is a counter indicating the repetitions that the $n$th pixel is grouped into the 3D cubes. Therefore, by taking this patchbased representation property into account, the traditional alternating direction algorithm can be derived to achieve fast computation. 


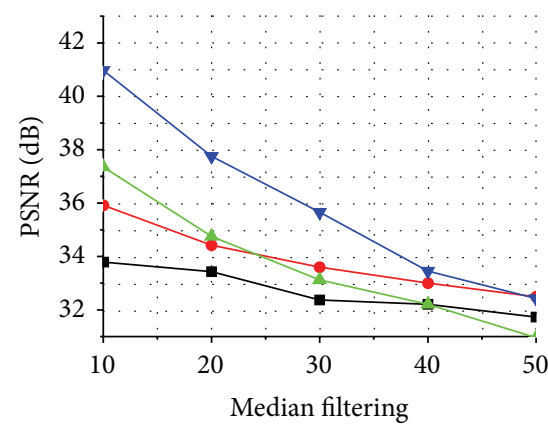

$\rightarrow$ Median filtering $\_$Wavelet $\rightarrow$ Total variation $\rightarrow$ PANO

(a)



(d)

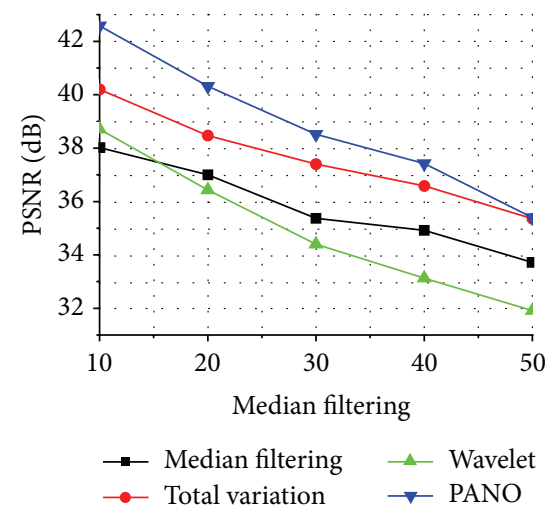

(b)

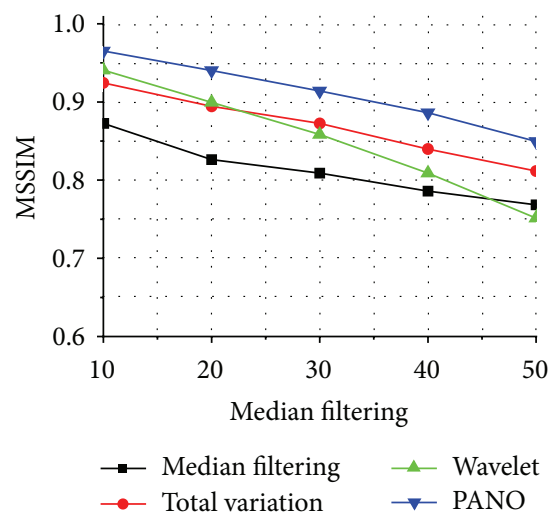

(e)

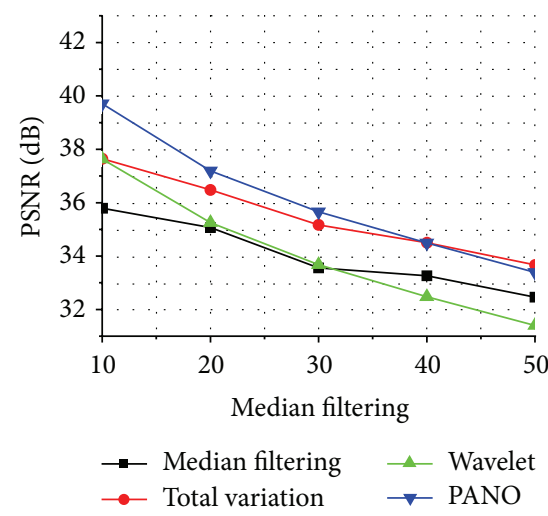

(c)

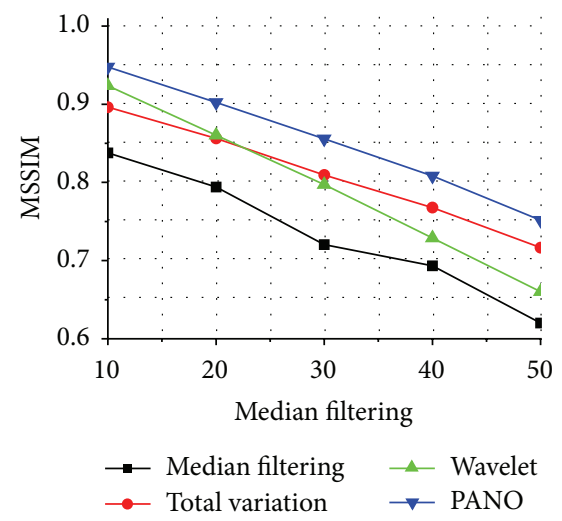

(f)

FIGURE 6: Denoising performance for salt-and-pepper noise. (a), (b), and (c) are the PSNRs of image Barbara, House, and Boat; (d), (e), and (f) are the MSSIMs of these images.

\section{Algorithm}

To numerically solve the optimization problem in (4), an alternating direction minimization with continuation (ADMC) algorithm $[30,31]$ is modified to remove the impulse noise. ADMC algorithm is also used for image deblurring $[32,33]$. Here, by introducing auxiliary variables $\boldsymbol{\alpha}_{j}=\mathbf{A}_{j} \mathbf{u}$ and $\mathbf{z}=\mathbf{f}-\mathbf{u}$ to (6), one has

$$
\begin{aligned}
\min _{\mathbf{u}, \boldsymbol{\alpha}, \mathbf{z}} \sum_{j=1}^{J}\left(\left\|\boldsymbol{\alpha}_{j}\right\|_{1}+\frac{\beta}{2}\left\|\boldsymbol{\alpha}_{j}-\mathbf{A}_{j} \mathbf{u}\right\|_{2}^{2}\right) \\
+\lambda\left(\|\mathbf{z}\|_{1}+\frac{\beta}{2}\|\mathbf{z}-(\mathbf{f}-\mathbf{u})\|_{2}^{2}\right) .
\end{aligned}
$$

As $\beta \rightarrow \infty$, the solution of (7) approaches that of (6). In practice, we use the previous solution as a "warm start" for the next alternating optimization as $\beta$ gradually increased. $\beta$ does not need to be very large for image denoising. Simulation results show that increasing it will not improve the quality of denoised image but need more computation time. Thus $\beta \leq 2^{14}$ is suggested. When $\beta$ is fixed, (7) can be solved in an alternating fashion between $\boldsymbol{\alpha}_{j}(j=1, \ldots, J), \mathbf{z}$, and $\mathbf{u}$. Consider the following.
(1) Fix $\mathbf{u}$ and $\mathbf{z}$; solve $\min _{\boldsymbol{\alpha}_{j}}\left\|\boldsymbol{\alpha}_{j}\right\|_{1}+(\beta / 2)\left\|\boldsymbol{\alpha}_{j}-\mathbf{A}_{j} \mathbf{u}\right\|_{2}^{2}(j=$ $1,2, \ldots, J)$, whose solution is

$$
\widehat{\boldsymbol{\alpha}}_{j}=S\left(\mathbf{A}_{j} \mathbf{u}, \frac{1}{\beta}\right), \quad(j=1,2, \ldots, J),
$$

where $S(\mathbf{v}, t)$ denotes the soft thresholding operation on $\mathbf{v}$ with threshold $t[30,31]$.

(2) Fix $\mathbf{u}$ and $\boldsymbol{\alpha}$; solve $\min _{\mathbf{z}}\|\mathbf{z}\|_{1}+(\beta / 2)\|\mathbf{z}-(\mathbf{f}-\mathbf{u})\|_{2}^{2}$, whose solution is

$$
\widehat{\mathbf{z}}=S\left(\mathbf{f}-\mathbf{u}, \frac{1}{\beta}\right) .
$$

(3) Fix $\mathbf{z}$ and $\boldsymbol{\alpha}$; solve $\min _{\mathbf{u}} \sum_{j=1}^{J}\left\|\boldsymbol{\alpha}_{j}-\mathbf{A}_{j} \mathbf{u}\right\|_{2}^{2}+$ $\lambda\|\mathbf{z}-(\mathbf{f}-\mathbf{u})\|_{2}^{2}$, whose solution is

$$
\widehat{\mathbf{u}}=(\lambda \mathbf{I}+\mathbf{O})^{-1}\left[\sum_{j=1}^{J} \mathbf{A}_{j}^{T} \boldsymbol{\alpha}_{j}+\lambda(\mathbf{f}-\mathbf{z})\right] .
$$

To construct PANO, similarity needs to be learnt so that some similar patches are grouped together and stacked into $3 \mathrm{D}$ cubes. However, the similarity is corrupted for a noisy image. To obtain a reasonable similarity, we need to have an 


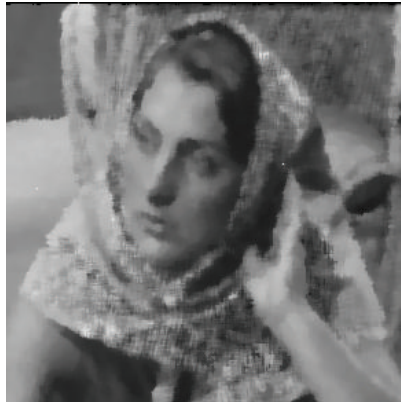

(a1)

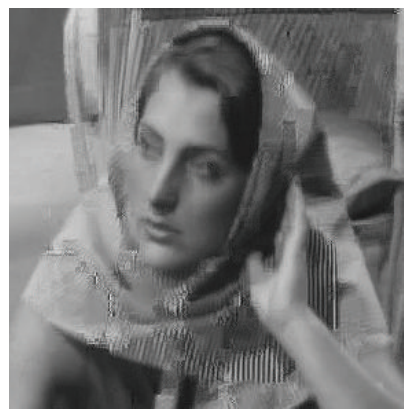

(a2)

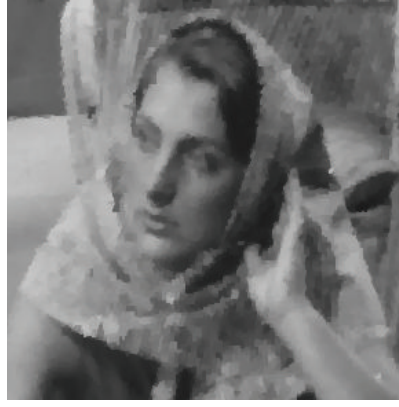

(b1)

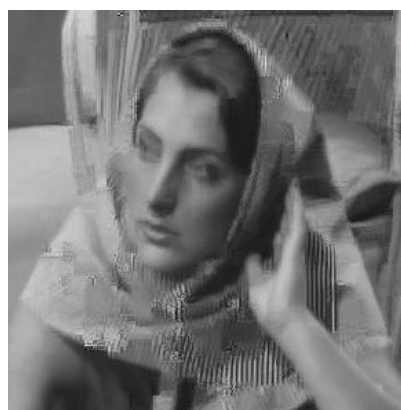

(b2)

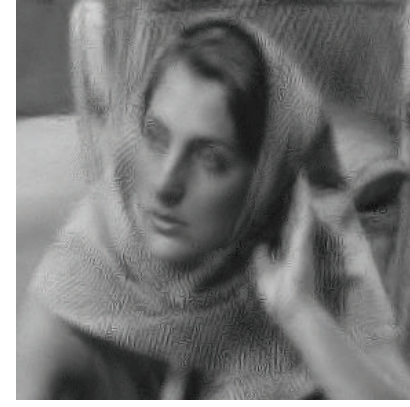

$(\mathrm{c} 1)$

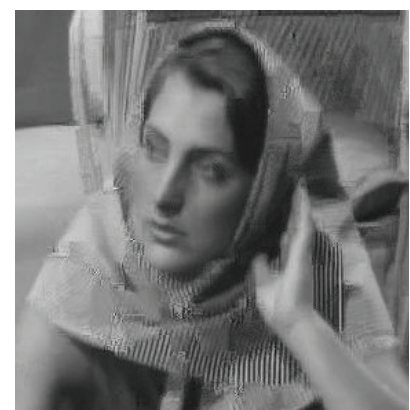

(c2)



(d1)

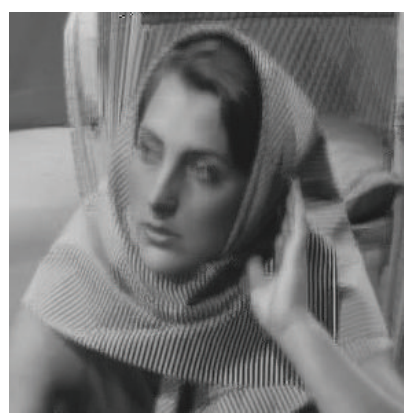

(d2)

Figure 7: Denoised Barbara images with 50\% salt-and-pepper noise. (al), (b1), and (cl) are initial guide image using median filtering, total variation, and wavelet, respectively; (d1) original image; (a2), (b2), (c2), and (d2) are denoising results of the proposed method using (a1), (b1), (cl), and (d1) as the initial guide image, respectively.

initial denoised image as a guide image. The similarity is first learnt from a denoised image using conventional methods such as median filtering and then learnt twice from the denoised image using PANO. The process of the modified algorithm is summarized as Algorithm 1.

\section{Results}

In simulations, the proposed PANO-based denoising method is compared with median filtering [1], wavelet-based sparse representation denoising, and total variation denoising. The dual-tree complex wavelet, which was used in impulse denoising [14], is adopted as the wavelet transform. PANO is applied with the typical parameter settings: the number of similar patches is 16 , the patch size is $8 \times 8$, and the search region is $39 \times 39$.

Objective image quality assessments, peak signal-tonoise ratio (PSNR), and mean measure of structural similarity (MSSIM) [34] are evaluated. The PSNR is defined as

$$
\operatorname{PSNR}(\mathrm{dB})=20 \log _{10} \frac{\sqrt{N}}{\|\mathbf{u}-\widehat{\mathbf{u}}\|},
$$

where $N$ is the number of pixels in an image and $\mathbf{u}$ and $\widehat{\mathbf{u}}$ are the original and denoised images, respectively. MSSIM is defined as

$$
\operatorname{MSSIM}=\frac{1}{N} \sum_{n=1}^{N} \operatorname{SSIM}\left(\mathbf{a}_{n}, \mathbf{b}_{n}\right)
$$

where $\mathbf{a}_{n}$ and $\mathbf{b}_{n}$ are the image content at the $n$th local window and SSIM evaluates local reconstruction errors by measuring the similarity between two images in a local window [34]. A higher MSSIM value means that structural information is better preserved and more suitable for human visual system.

The experiments run on 4 Cores $2.6 \mathrm{GHz} \mathrm{CPU}$ laptop computer with $12 \mathrm{~GB}$ RAM. The computational time of the proposed method is approximately 25 (or 70) seconds for image with size $256 \times 256$ (or $512 \times 512$ ). For the simulated noisy images, the window size of the median filtering and the regularization parameters of the rest methods are optimized to maximize MSSIMs while removing most of the impulse noise.

4.1. Choose Guide Images. The similarity is assumed to be known in the proposed denoising model. However, the similarity is also corrupted for a noisy image. To obtain a reasonable similarity, we need to have an initial denoised image as a guide image. In this section, we will discuss how to choose a guide image.

Since conventional denoising methods are available, one may consider taking the denoising result by median filtering, total variation, or wavelet denoising as the guide image to learn the similarity. When a better denoised image is obtained using the proposed approach, one may further utilize the result as an updated guide image.

As shown in Figure 3, different initial guide images lead to comparable denoising results with $30 \%$ noise. So median 


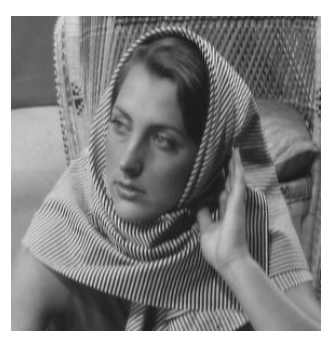

(a1)



(a2)



(a3)

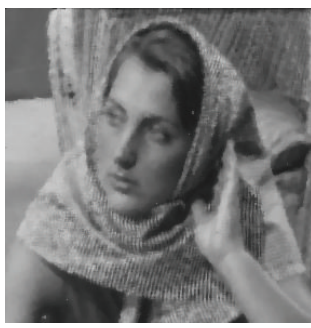

(b1)

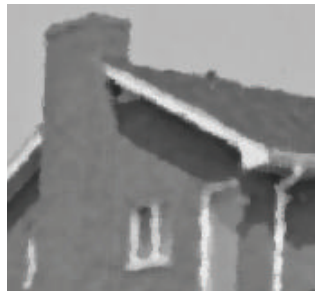

(b2)

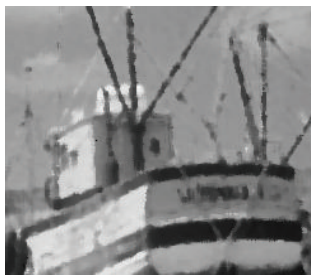

(b3)

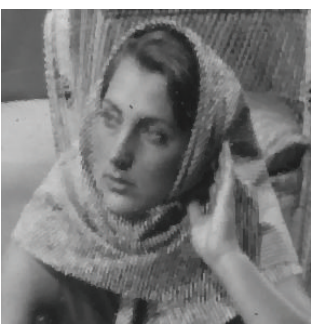

(c1)

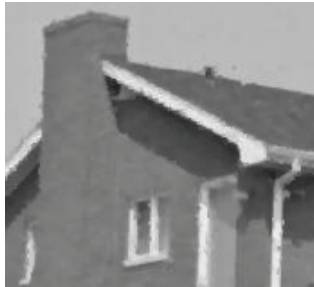

(c2)

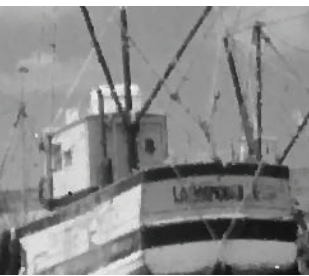

(c3)

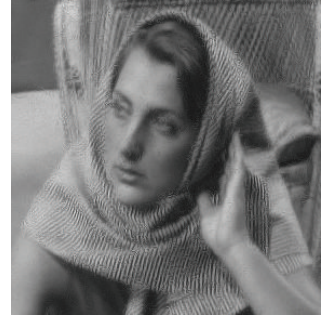

(d1)

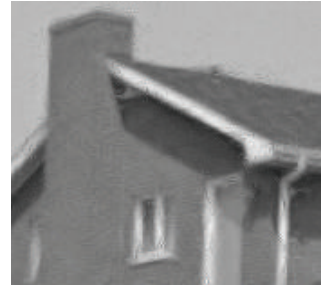

(d2)

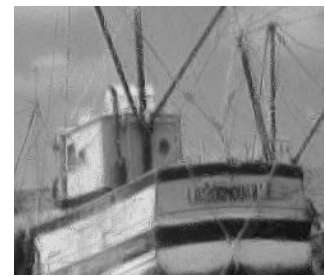

(d3)



(e1)

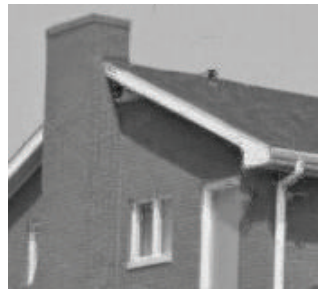

(e2)



(e3)

FIGURE 8: Denoised images with 30\% random-valued impulse noise. (a1), (a2), and (a3) are original images; (b1), (b2), and (b3) are results of median filtering; (c1), (c2), and (c3) are results of total variation denoising; (d1), (d2), and (d3) results of the wavelet-based sparse representation denoising; (e1), (e2), and (e3) are results of the proposed method.

filtering is chosen to produce the initial guide image since its computation is the fastest. After the similarity is learnt for 3 times, both the PSNR and MSSIM of the proposed method become stable, and more similarity learning will cost more computation time. Hence, learning the similarity 3 times is sufficient.

4.2. Remove Simulated Impulse Noise. Images (Figures 3(a), $4(\mathrm{a})$, and $4(\mathrm{~d})$ ), which are usually used in image processing, are treated as ground truth images; salt-and-pepper noise or random-valued impulse noise is manually added in simulations.

4.2.1. Salt-and-Pepper Noise Removal. The performances of the four denoising methods for salt-and-pepper noise are compared. As shown in Figure 5, repeated image patterns, for example, textures in Barbara, are obviously lost using median filtering and total variation. Although wavelet recovers the textures much better than the above two methods, it suffers from losing edges. The proposed method preserves these features best, with the clearest textures in Barbara and edges in both House and Boat.

As shown in Figure 6, the proposed method achieves the highest PSNRs when the noise level is below $40 \%$ and the highest SSIMs for all noise levels. When the noise becomes heavier (e.g., 50\%), the performance of the proposed method gradually dropped. As Figure 7 shows, the qualities of the initial guide images using median filtering, total variation, and wavelet denoising methods are all unsatisfactory; thus, similarity cannot be learnt well so that the denoising results of the proposed method are also not satisfactory. Table 1 shows the performance of the proposed method using different initial guide images under heavy noise. Suppose we have the original image as the initial guide image so that similarity information can be exactly leant; the proposed method can remove the noise very well and preserve the image structures. In summary, the proposed method leads to comparable denoising results when ground truth image is not available.

4.2.2. Random-Valued Impulse Noise Removal. The performances of the four denoising methods for random-valued impulse noise are also compared. As shown in Figure 8, edges and textures are best preserved by the proposed method. Under different noise levels, proposed method achieves the highest MSSIMs (Figure 9). These observations imply that the proposed method preserves the best image structures during removing the random-valued impulse noise. 


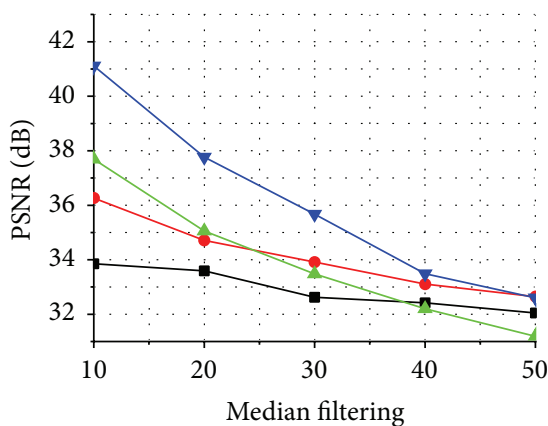

$\rightarrow$ Median filtering $\_$Wavelet $\rightarrow$ Total variation $\rightarrow$ PANO

(a)

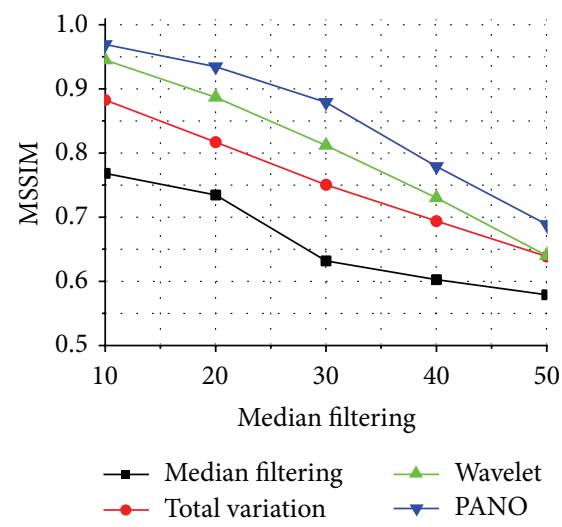

(d)



$\rightarrow$ Median filtering $\quad \_$Wavelet $\rightarrow$ Total variation $\rightarrow$ PANO

(b)

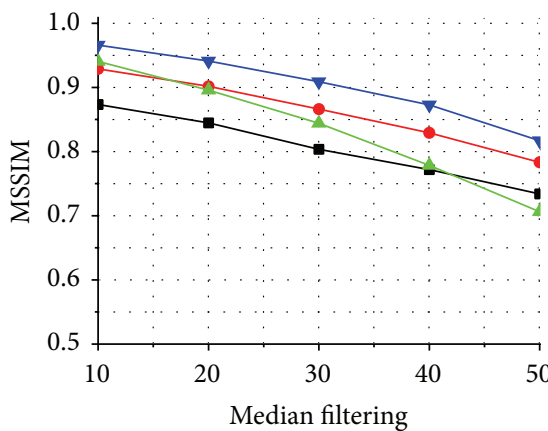

$\rightarrow$ Median filtering $\longrightarrow$ Wavelet

(e)

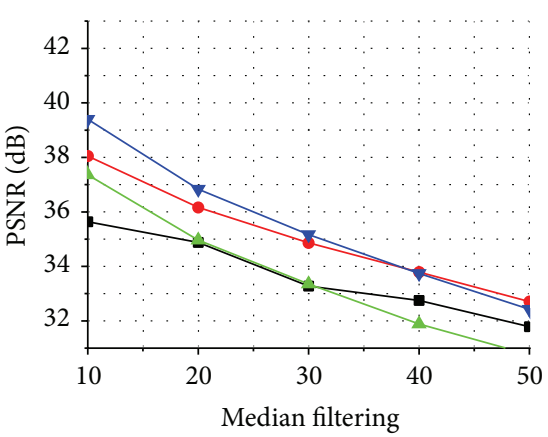

$\rightarrow$ Median filtering $\longrightarrow$ Wavelet $\rightarrow$ Total variation $\rightarrow$ PANO

(c)

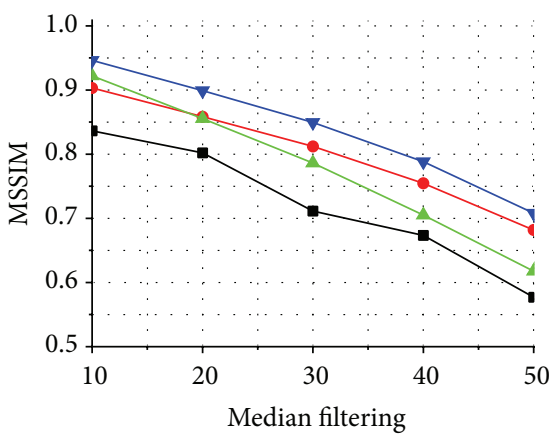

$\rightarrow$ Median filtering $\rightarrow$ Wavelet

(f)

FIGURE 9: Denoising performance for random-valued impulse noise. (a), (b), and (c) are the PSNRs of image Barbara, House, and Boat; (d), (e), and (f) are the MSSIMs of these images.

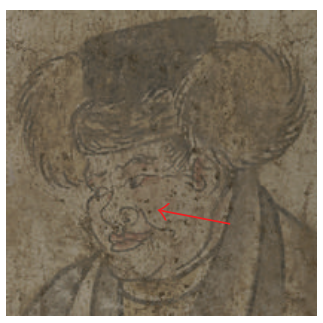

(a1)



(a2)

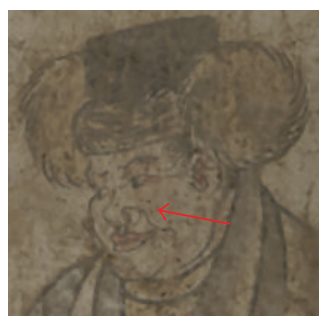

(b1)

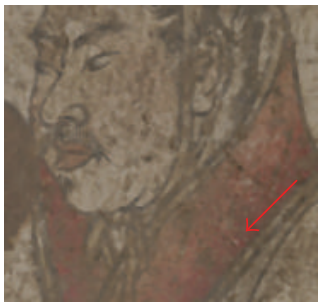

(b2)

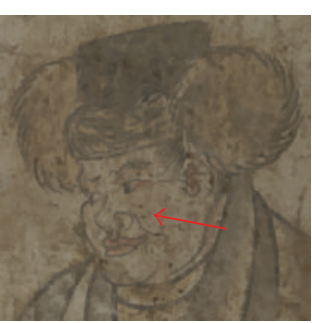

(c1)

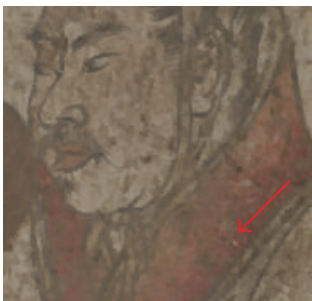

(c2)

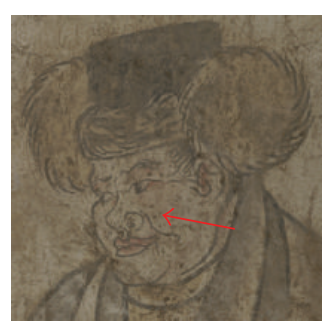

(d1)

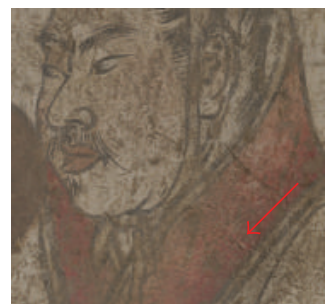

(d2)



(e1)

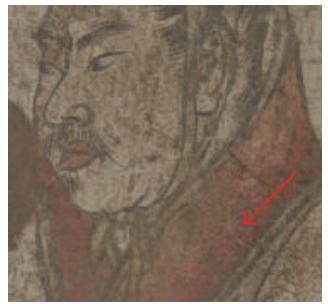

(e2)

FIGURE 10: Mural images after removing impulse noise-like artifacts. (a1), (a2) are original images; (b1), (b2) are results of median filtering; (c1), (c2) are results of total variation denoising; (d1), (d2) are results of the wavelet-based sparse representation denoising; (e1), (e2) are results of the proposed method. 


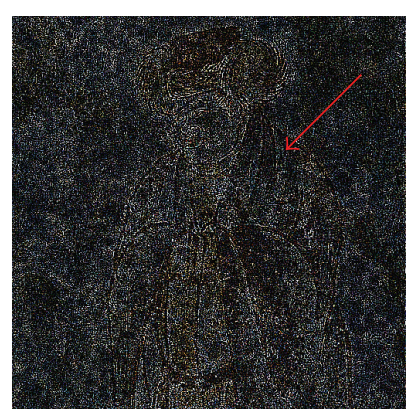

(a1)



(a2)

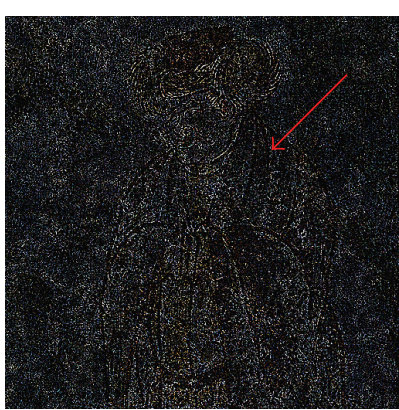

(b1)

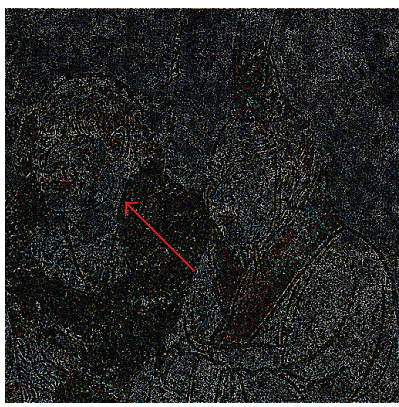

(b2)



(c1)

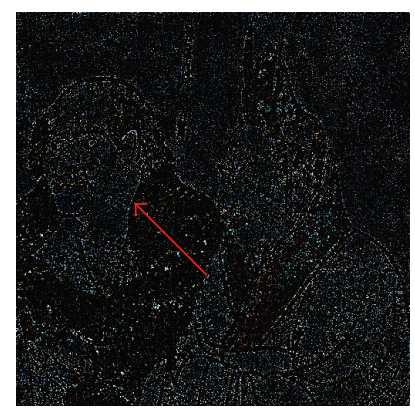

(c2)

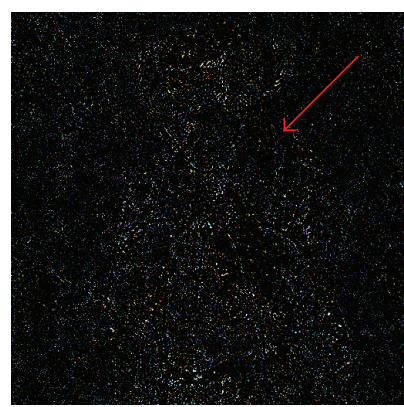

(d1)

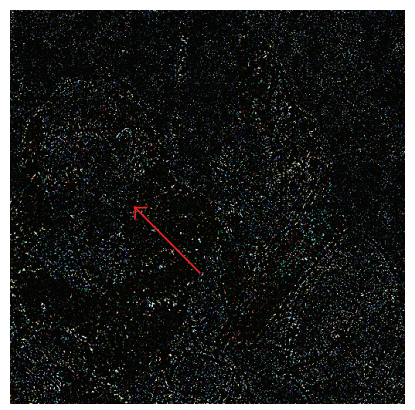

(d2)

FiguRE 11: Difference between original and denoised images. (a1), (b1), (c1), and (d1) are lost structures in denoising Figure 1(b) using median filtering, total variation, wavelet, and the proposed method, respectively; (a2), (b2), (c2), and (d2) are lost structures in denoising Figure 1(c) using median filtering, total variation, wavelet, and the proposed method, respectively.

4.3. Remove Impulse Noise-Like Artifacts in Mural Images. In the section, the impulse noise-like artifacts in realistic mural images are suppressed using these denoising methods. As shown in Figure 10, both median filtering and total variation will easily blur the images. On the contrary, the proposed method preserves the image details much better and suppresses most the impulse noise-like artifacts as marked. We can observe that the lost image structures are minimal among these methods (Figure 11). Therefore, the proposed method can also be used to remove these impulse noise-like artifacts of real dataset.

4.4. The Effect of Noise Detectors. Noise detectors are very useful to improve the denoising performance of salt-andpepper noise. Chan et al. [18] proposed to use a noise detector first and apply a weighted regularization model with less penalization on the noise-free pixels. Following this idea, we use a noise detector to distinguish the noisy pixels from the rest of the pixels in the preliminary results. The locations of detected noise candidates are stored in a set $\Omega$, whose complementary set $\bar{\Omega}$ stores locations of the rest of the pixels. We tried a weighted $l_{1}-l_{1}$ regularization model as

$$
\min _{\mathbf{u}}\left\{\|\Psi \mathbf{u}\|_{1}+\lambda\|\mathbf{W}(\mathbf{f}-\mathbf{u})\|_{1}\right\}
$$

where $\mathbf{W}$ is a diagonal matrix, whose $m$ th diagonal entry is $w_{m}$. A small weight $w_{i}(i \in \Omega)$ is assigned for noise candidates and a large weight $w_{k}(k \in \bar{\Omega})$ is set for the rest of pixels. $\Psi$ is chosen to be total variation or PANO.

Simulation results listed in Table 2 show that noise detectors can significantly improve the denoising performance. When both methods are equipped with noise detectors, the proposed method still outperforms total variation with higher MSSIMs and comparable PSNRs. This implies that the proposed method can preserve image structures better, as shown in Figure 12.

An efficient method to suppress the noise via combining PANO with noise detectors can be found in [35]. However, the noise detectors proposed in [18] only work for salt-andpepper noise. How to detect the noise candidates in randomvalued noisy image is still unknown. Besides, how to solve the proposed weighted regularization model efficiently and setting the weight optimally need comprehensive investigation. We leave these as the future work.

\section{Conclusions}

Impulse noise removal with similarity-motivated sparse representation is proposed. With a patch-based nonlocal operator to model sparse representation of similar image patches, the $l_{1}-l_{1}$ optimization problem is solved by a modified iterative alternating direction minimization algorithm.

Experiments on simulated data demonstrate that the proposed method significantly improve the recovery of textures and edges while removing both salt-and-pepper noise 


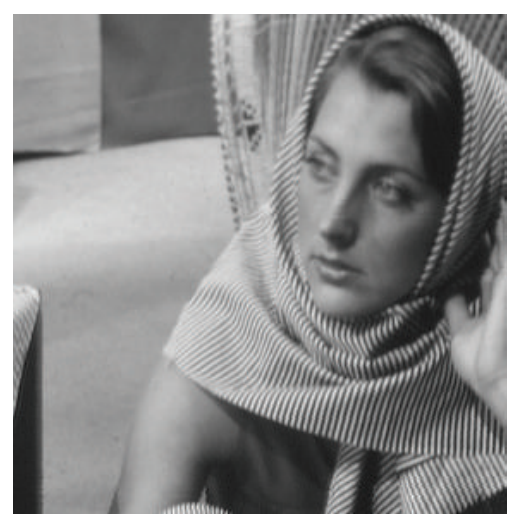

(a)



(b)

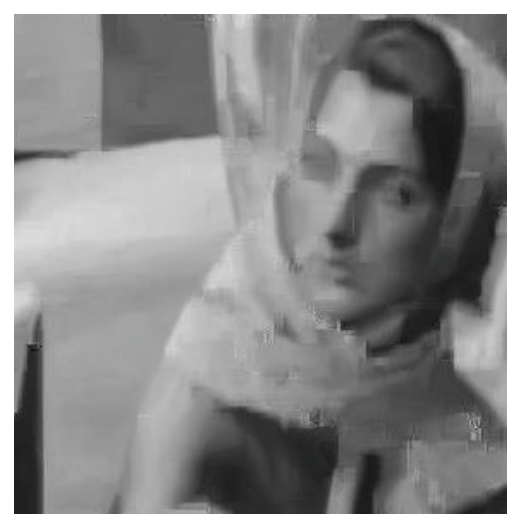

(c)

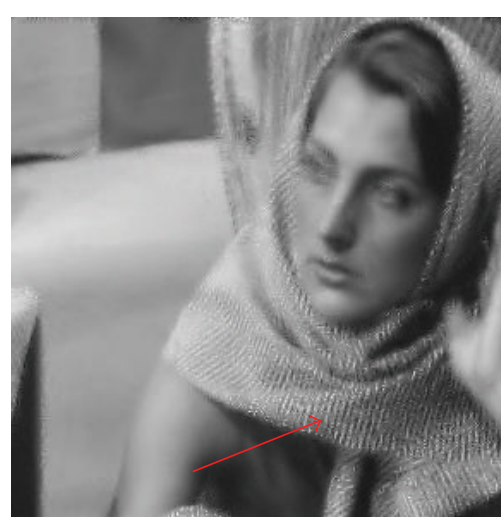

(d)

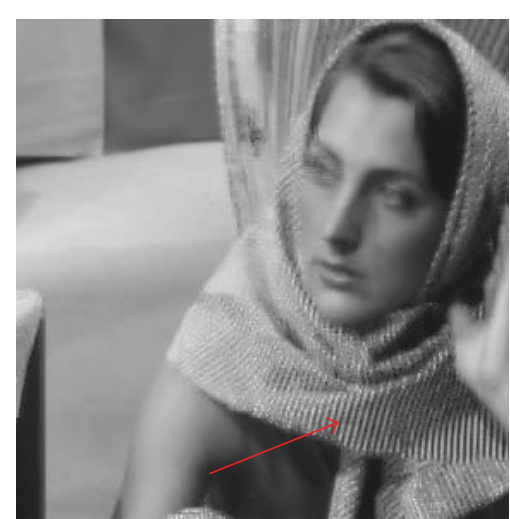

(e)

Figure 12: Denoising results of Barbara image with 70\% salt-and-pepper noise. (a) Ground truth image, (b) noisy image, and (c), (d), and (e) are denoised images using the proposed method, total variation with noise detectors, and the proposed method with noise detectors, respectively.

and random-valued impulse noise. It can be also used to remove the impulse noise-like artifacts in mural images well. Denoising performance of the proposed method may be further improved if one can learn a proper similarity from serious noisy image.

\section{Conflict of Interests}

The authors declare that there is no conflict of interests regarding the publication of this paper.

\section{Acknowledgments}

This work was supported in part by the National Natural Science Foundation of China (61302174, 61201045), Scientific Research Foundation for the Introduction of Talent at Xiamen University of Technology (YKJ12021R), Open Fund from Key Lab of Digital Signal and Image Processing of Guangdong Province (2013GDDSIPL-07, 54600321), Fundamental Research Funds for the Central Universities (2013SH002), and Natural Science Foundation of Shaanxi Province of China (2013JM8015).

\section{References}

[1] R. C. Gonzalez and E. Richard, Digital Image Processing, Prentice-Hall, 2002.

[2] M. Elad and M. Aharon, "Image denoising via sparse and redundant representations over learned dictionaries," IEEE Transactions on Image Processing, vol. 15, no. 12, pp. 3736-3745, 2006.

[3] L. Jacques, L. Duval, C. Chaux, and G. Peyré, "A panorama on multiscale geometric representations, intertwining spatial, directional and frequency selectivity," Signal Processing, vol. 91, no. 12, pp. 2699-2730, 2011.

[4] L. Bao, M. Robini, W. Liu, and Y. Zhu, "Structure-adaptive sparse denoising for diffusion-tensor MRI," Medical Image Analysis, vol. 17, no. 4, pp. 442-457, 2013.

[5] D. Huang, L. Kang, Y. Wang, and C. Lin, "Self-learning based image decomposition with applications to single image denoising," IEEE Transactions on Multimedia, vol. 16, pp. 83-93, 2014.

[6] J. Starck, M. Elad, and D. L. Donoho, "Image decomposition via the combination of sparse representations and a variational approach," IEEE Transactions on Image Processing, vol. 14, no. 10, pp. 1570-1582, 2005.

[7] L.-W. Kang, C.-W. Lin, and Y.-H. Fu, "Automatic single-imagebased rain streaks removal via image decomposition," IEEE 
Transactions on Image Processing, vol. 21, no. 4, pp. 1742-1755, 2012.

[8] P. Wang, H. Tian, and W. Zheng, "A novel image fusion method based on FRFT-NSCT," Mathematical Problems in Engineering, vol. 2013, Article ID 408232, 9 pages, 2013.

[9] L. Xu, J. Du, and Q. Li, "Image fusion based on nonsubsampled contourlet transform and saliency-motivated pulse coupled neural networks," Mathematical Problems in Engineering, vol. 2013, Article ID 135182, 10 pages, 2013.

[10] J. Wright, A. Y. Yang, A. Ganesh, S. S. Sastry, and Y. Ma, "Robust face recognition via sparse representation," IEEE Transactions on Pattern Analysis and Machine Intelligence, vol. 31, no. 2, pp. 210-227, 2009.

[11] H. Cheng, Z. Liu, L. Yang, and X. Chen, "Sparse representation and learning in visual recognition: theory and applications," Signal Processing, vol. 93, no. 6, pp. 1408-1425, 2013.

[12] C. Lin, B. H. Wang, X. F. Zhao, and M. Pang, "Optimizing kernel PCA using sparse representation-based classifier for mstar SAR image target recognition," Mathematical Problems in Engineering, vol. 2013, Article ID 847062, 10 pages, 2013.

[13] H. Cheng, Z. Liu, L. Hou, and J. Yang, "Sparsity induced similarity measure and its applications," IEEE Transactions on Circuits and Systems for Video Technology, no. 99, 1 page, 2013.

[14] S. Huang and J. Zhu, "Removal of salt-and-pepper noise based on compressed sensing," Electronics Letters, vol. 46, no. 17, pp. 1198-1199, 2010.

[15] Q. Liu, S. Wang, J. Luo, Y. Zhu, and M. Ye, "An augmented Lagrangian approach to general dictionary learning for image denoising," Journal of Visual Communication and Image Representation, vol. 23, no. 5, pp. 753-766, 2012.

[16] S. Wang, Q. Liu, Y. Xia et al., "Dictionary learning based impulse noise removal via L1-L1 minimization," Signal Processing, vol. 93, no. 9, pp. 2696-2708, 2013.

[17] M. Nikolova, "A variational approach to remove outliers and impulse noise," Journal of Mathematical Imaging and Vision, vol. 20, no. 1-2, pp. 99-120, 2004.

[18] R. H. Chan, H. Chung-Wa, and M. Nikolova, "Salt-andpepper noise removal by median-type noise detectors and detail-preserving regularization," IEEE Transactions on Image Processing, vol. 14, no. 10, pp. 1479-1485, 2005.

[19] J. Cai, R. H. Chan, and C. Di Fiore, "Minimization of a detail-preserving regularization functional for impulse noise removal," Journal of Mathematical Imaging and Vision, vol. 29, no. 1, pp. 79-91, 2007.

[20] J. Cai, R. H. Chan, and M. Nikolova, "Fast two-phase image deblurring under impulse noise," Journal of Mathematical Imaging and Vision, vol. 36, no. 1, pp. 46-53, 2010.

[21] X.-G. Lv, J. Le, J. Huang, and L. Jun, "A fast high-order total variation minimization method for multiplicative noise removal," Mathematical Problems in Engineering, vol. 2013, Article ID 834035, 13 pages, 2013.

[22] Y. Shi and Q. Chang, "Efficient algorithm for isotropic and anisotropic total variation deblurring and denoising," Journal of Applied Mathematics, vol. 2013, Article ID 797239, 14 pages, 2013.

[23] A. Buades, B. Coll, and J. M. Morel, "A review of image denoising algorithms, with a new one," Multiscale Modeling and Simulation, vol. 4, no. 2, pp. 490-530, 2005.

[24] K. Dabov, A. Foi, V. Katkovnik, and K. Egiazarian, "Image denoising by sparse 3-D transform-domain collaborative filtering," IEEE Transactions on Image Processing, vol. 16, no. 8, pp. 2080-2095, 2007.
[25] J. V. Manjón, P. Coupé, A. Buades, D. Louis Collins, and M. Robles, "New methods for MRI denoising based on sparseness and self-similarity," Medical Image Analysis, vol. 16, no. 1, pp. 1827, 2012.

[26] Y. K. Hou, C. X. Zhao, D. Y. Yang, and Y. Cheng, "Comments on image denoising by sparse 3-D transform-domain collaborative filtering," IEEE Transactions on Image Processing, vol. 20, no. 1, pp. 268-270, 2011.

[27] X. Qu, Y. Hou, F. Lam, D. Guo, J. Zhong, and Z. Chen, "Magnetic resonance image reconstruction from undersampled measurements using a patch-based nonlocal operator," Medical Image Analysis, vol. 18, pp. 843-856, 2014.

[28] P. Rodriguez, "Total variation regularization algorithms for images corrupted with different noise models: a review," Journal of Electrical and Computer Engineering, vol. 2013, Article ID 217021, 18 pages, 2013.

[29] X. Qu, Y. Hou, F. Lam, D. Guo, and Z. Chen, "Magnetic resonance image reconstruction using similarities learnt from multi-modal images," in Proceedings of the IEEE China Summit and International Conference on Signal and Information Processing (ChinaSIP '13), pp. 264-268, IEEE, Beijing, China, 2013.

[30] J. Yang, Y. Zhang, and W. Yin, "A fast alternating direction method for TVL1-L2 signal reconstruction from partial Fourier data," IEEE Journal on Selected Topics in Signal Processing, vol. 4, no. 2, pp. 288-297, 2010.

[31] X. Qu, D. Guo, B. Ning et al., "Undersampled MRI reconstruction with patch-based directional wavelets," Magnetic Resonance Imaging, vol. 30, no. 7, pp. 964-977, 2012.

[32] Y. M. Huang, M. K. Ng, and Y. W. Wen, "A fast total variation minimization method for image restoration," Multiscale Modeling \& Simulation, vol. 7, no. 2, pp. 774-795, 2008.

[33] X. X. Guo, F. Li, and M. K. Ng, "A fast $l_{1}-\mathrm{TV}$ algorithm for imgage restoration," SIAM Journal on Scientific Computing, vol. 31, pp. 2322-2341, 2009.

[34] Z. Wang, A. C. Bovik, H. R. Sheikh, and E. P. Simoncelli, "Image quality assessment: from error visibility to structural similarity," IEEE Transactions on Image Processing, vol. 13, no. 4, pp. 600612, 2004.

[35] D. Guo, X. Qu, X. Du, K. Wu, and X. Chen, "Salt and pepper noise removal with noise detection and a patch-based sparse representation," Advances in Multimedia, vol. 2014, Article ID 682747, 14 pages, 2014. 




Advances in

Operations Research

mansans



The Scientific World Journal
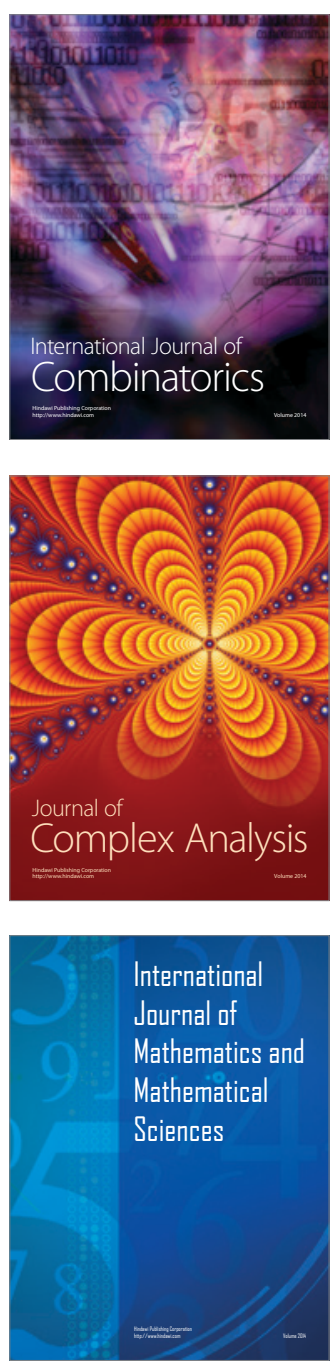
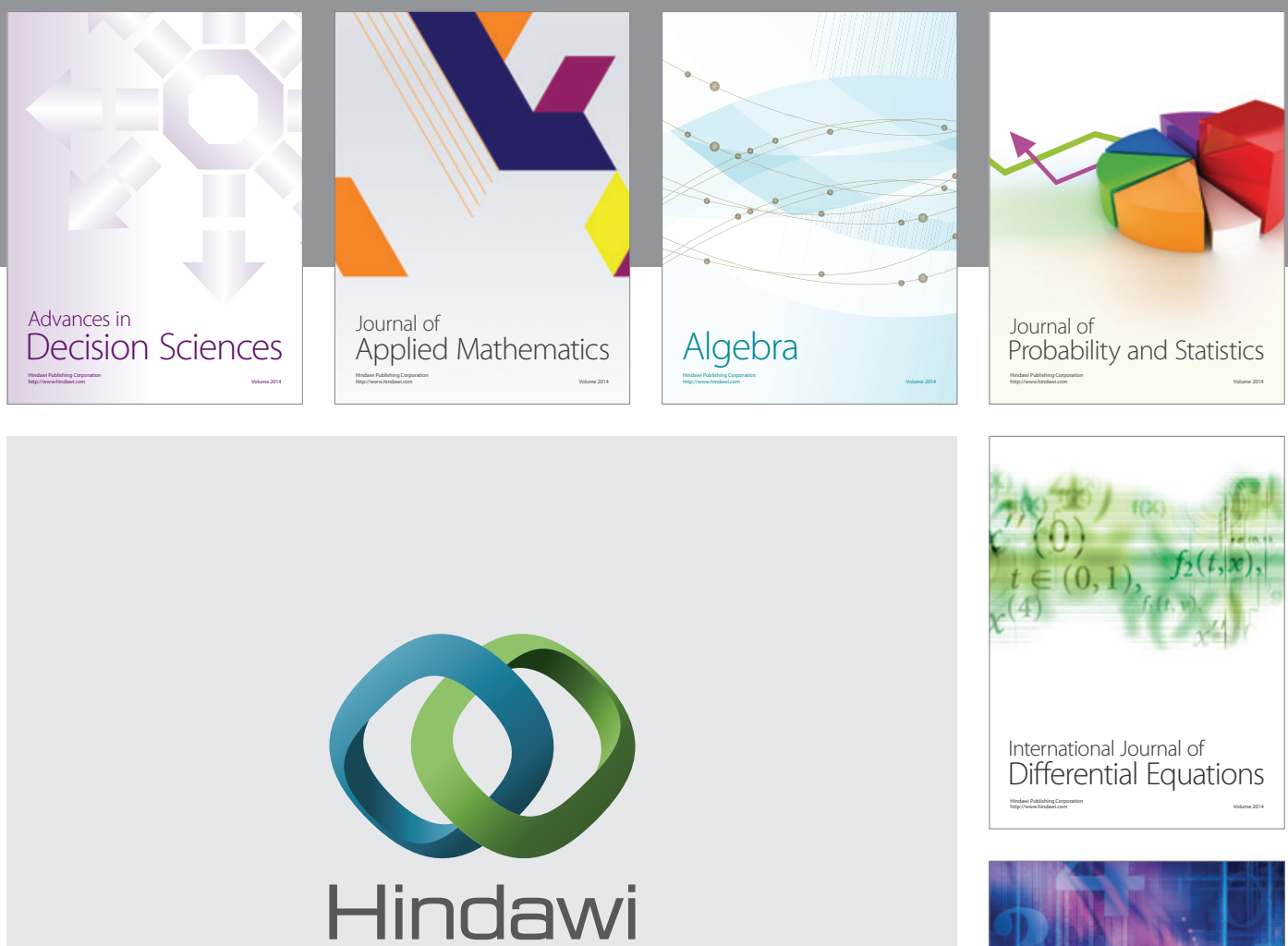

Submit your manuscripts at http://www.hindawi.com
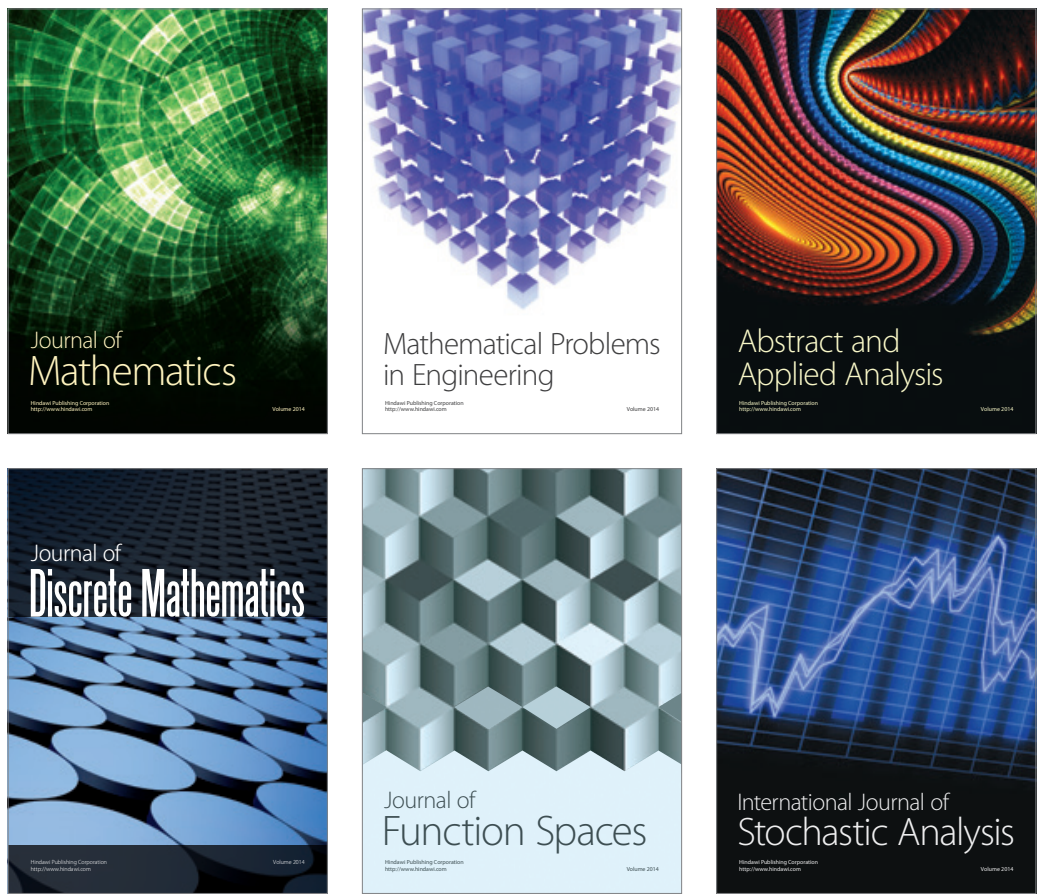

Journal of

Function Spaces

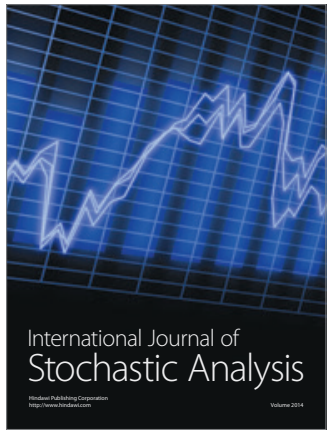

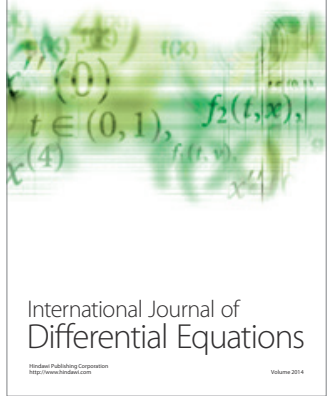
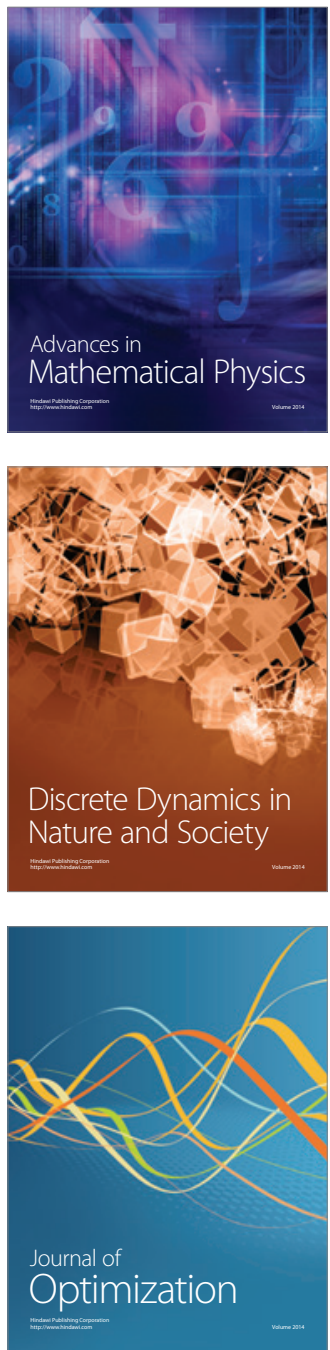